\title{
Absorção de nutrientes e distribuição da massa fresca e seca entre órgãos de batata-doce
}

\author{
Fábio R Echer²; Júlio C Dominato²; José E Creste ${ }^{1}$ \\ ${ }^{1}$ UNOESTE-CCA, Rod. Raposo Tavares, km 572, 19067-175 Presidente Prudente-SP; ${ }^{2}$ Mestrando em Agronomia; \\ fabioecher@gmail.com
}

\begin{abstract}
RESUMO
A marcha de absorção estuda a relação existente entre a quantidade de nutrientes, o acúmulo de massa seca e a idade da planta. $\mathrm{O}$ objetivo deste trabalho foi determinar a marcha de absorção de nutrientes e a produção de massa fresca e seca dos órgãos da batatadoce, cultivar Canadense. O experimento foi conduzido entre os meses de agosto e dezembro de 2007, em lavoura comercial, em Presidente Prudente-SP. O delineamento experimental utilizado foi inteiramente casualizado, com três repetições. Os tratamentos consistiram em oito épocas de coleta iniciadas aos 40 dias após o transplantio, com intervalo entre coletas de 15 dias. As raízes tuberosas proporcionaram maior produção de massa fresca e seca no momento da colheita em relação aos demais órgãos. $\mathrm{O} \mathrm{N}$ e o $\mathrm{Mn}$ foram os macro e micronutrientes mais absorvidos pelas folhas, ramas e raízes tuberosas de batata-doce, enquanto na raiz, observou-se maior absorção de $\mathrm{Ca}$ e Mn. O período de maior absorção de $\mathrm{N}$ e K pelas raízes tuberosas foi entre 115-145 e 115-130 dias após o transplantio, respectivamente.
\end{abstract}

Palavras-chave: Ipomoea batatas, extração de nutrientes, produção de biomassa.

\begin{abstract}
Absorption march of nutrients and distribution of fresh and dry matter among organs of sweet-potato

The absorption march studies the relationship between the amount of nutrients, dry mass accumulation and the plant age. The absorption march of nutrients, fresh mass and dry mass production of sweet potato organs was determined on the cultivar Canadense. The experiment was carried out between August and December, 2007, in Presidente Prudente, São Paulo State, Brazil, on a commercial farm. The completely randomized experimental design was adopted, with three repetitions. The treatments consisted of eight collection dates, beginning 40 days after transplanting, at 15-day intervals. The storage roots provided higher production of fresh and dry mass at harvest in comparison to other organs. $\mathrm{N}$ and $\mathrm{Mn}$ were the macro and micronutrients more absorbed by leaves, stems and storage roots of sweet potato, while higher absorption of $\mathrm{Ca}$ and $\mathrm{Mn}$ was observed in the roots. The period of larger absorption of $\mathrm{N}$ and $\mathrm{K}$ by storage roots was between 115-145 and 115-130 days after transplanting date, respectively.
\end{abstract}

Keywords: Ipomoea batatas; nutrient extraction, biomass yield.

(Recebido para publicação em 28 de maio de 2008; aceito em 23 de abril de 2009) (Received in May 28, 2008; accepted in April 23, 2009)

\begin{abstract}
A marcha de absorção estuda a relação existente entre a quantidade de nutrientes, $o$ acúmulo de matéria seca e a idade da planta, permitindo identificar, a) quantidade de nutrientes necessários para a produção; b) época de maior exigência de cada nutriente; c) em qual órgão cada nutriente se encontra em maior quantidade; d) o quanto é exportado pela colheita e o quanto será necessário repor ao solo para não exauri-lo (Marschner, 1995). Portanto, a marcha de absorção das culturas é uma importante ferramenta para auxiliar os programas de adubação e o manejo de fertilizantes das lavouras. No entanto, no Brasil, informações sobre a absorção de nutrientes e a curva de acúmulo de massa seca na cultura da batata-doce são escassos ou inexistentes.
\end{abstract}

A composição mineral nos tecidos foliares varia conforme a espécie, culti- var, tipo de folha e idade da planta (Malavolta et al., 1997), e a absorção de nutrientes em hortaliças segue um padrão de crescimento ou acúmulo de massa seca, sendo o potássio normalmente o mais absorvido (Ferreira et al., 1990).

A batata-doce é uma dicotiledônea da família Convolvulaceae, que possui alta eficiência na captação da energia solar e grande capacidade de produzir matéria seca por um longo período de tempo (Hahn, 1977). Seu caule é herbáceo de hábito prostrado, folhas largas e pecíolo longo (Embrapa, 2008). Possui dois tipos de raiz: a tuberosa, que constitui a principal parte de interesse comercial, e a raiz absorvente, responsável pela absorção de água e extração de nutrientes do solo.

Os mecanismos morfo-fisiológicos contribuem para o uso eficiente de nutrientes pelas plantas. Entre eles, estão o sistema radicular eficiente e extensivo; alta relação raiz/parte aérea; associação a microrganismos que fixam $\mathrm{N}_{2}$ atmosférico; habilidade das raízes em modificar a rizosfera para superar situações de baixa disponibilidade de nutrientes; capacidade de manter o metabolismo inalterado com baixas concentrações de nutrientes nos tecidos e, alta taxa fotossintética (Fageria \& Baligar, 1993).

Por ter elevado potencial produtivo, a batata-doce requer grande quantidade de nutrientes. Para uma produção de 11 a $15 \mathrm{t} \mathrm{ha}^{-1}$ de raízes tuberosas, estimase que a extração seja de 60 a $113 \mathrm{~kg}$ de $\mathrm{N}, 20$ a $47,5 \mathrm{~kg}$ de $\mathrm{P}_{2} \mathrm{O}_{5}, 100$ a $236 \mathrm{~kg}$ de $\mathrm{K}_{2} \mathrm{O}, 31$ a $35 \mathrm{~kg}$ de $\mathrm{CaO}$ e 11 a $13 \mathrm{~kg}$ de $\mathrm{MgO}$ (Silva et al., 2002).

No Brasil, dados referentes à extração de micronutrientes pela batata-doce são escassos. No entanto, relatos na literatura indicam que para cada tonelada 
de raízes tuberosas de batata-doce, são extraídas 66 gramas de B (Byju, 2007), assim para produtividades de $15 \mathrm{t} \mathrm{ha}^{-1}$, a exportação seria em torno de $1 \mathrm{~kg}$ de $\mathrm{B}$.

Scott (1950) estudou a absorção de potássio pela batata-doce; nos primeiros 60 dias a absorção foi baixa, aumentando aos 90 dias, sendo que quase todo potássio foi acumulado na parte aérea da planta, havendo translocação para as raízes tuberosas aos 120 e 150 dias.

Em outro trabalho realizado por Fabro et al. (1976), concluiu-se que a absorção de nutrientes em batata-doce varia significativamente com o tempo, com destaque para o potássio e o nitrogênio que a partir da oitava semana após o plantio tiveram altas taxas de absorção.

O objetivo deste trabalho foi determinar a marcha de absorção de nutrientes e a produção de massa seca dos órgãos da batata-doce em lavoura comercial no município de Presidente Prudente, oeste paulista.

\section{MATERIAL E MÉTODOS}

O experimento foi realizado entre os meses de agosto e dezembro de $2007 \mathrm{em}$ lavoura comercial de batata doce, no Rancho Ebenezer, em Mundo Novo, Distrito de Montalvão, Presidente Prudente-SP, definida pelas coordenadas geográficas: latitude $22^{\circ} 06^{\prime} 50$ " $\mathrm{S}$; longitude $51^{\circ} 27^{\prime} 32^{\prime \prime} \mathrm{W}$ e $406 \mathrm{~m}$ de altitude. Na figura 1 estão os dados referentes às condições climáticas durante a condução do experimento.

O solo da área foi classificado como Argissolo Vermelho-Amarelo arenoso (Embrapa, 1999). A análise do solo revelou as características: $\mathrm{pH}=5,4\left(\mathrm{CaCl}_{2} 0,01\right.$ $\left.\mathrm{mol} \mathrm{L}^{-1}\right) ; \mathrm{MO}=10 \mathrm{~g} \mathrm{dm}^{-3} ; \mathrm{P}_{\text {resina }}=39 \mathrm{mg}$ $\mathrm{dm}^{-3} ; \mathrm{H}+\mathrm{Al}=15 \mathrm{mmol} \mathrm{dm}^{-3} ; \mathrm{K}=2,1 \mathrm{mmol}_{\mathrm{c}}$ $\mathrm{dm}^{-3} ; \mathrm{Ca}=19 \mathrm{mmol}_{\mathrm{c}} \mathrm{dm}^{-3} ; \mathrm{Mg}^{-}=5 \mathrm{mmol}_{\mathrm{c}}{ }^{\mathrm{c}}$ $\mathrm{dm}^{-3} ; \mathrm{S}=5,6 \mathrm{mg} \mathrm{dm}^{-3} ; \mathrm{SB}=26 \mathrm{mmol}_{\mathrm{c}} \mathrm{dm}^{-3}$; $\mathrm{CTC}=41 \mathrm{mmol}_{\mathrm{c}} \mathrm{dm}^{-3}$; saturação por ba$\mathrm{ses}=63 \% ; \mathrm{B}=0,13 \mathrm{mg} \mathrm{dm}^{-3} ; \mathrm{Cu}=0,7 \mathrm{mg}$ $\mathrm{dm}^{-3} ; \mathrm{Fe}=19 \mathrm{mg} \mathrm{dm}^{-3} ; \mathrm{Mn}=40,4 \mathrm{mg} \mathrm{dm}^{-3}$ e $\mathrm{Zn}=1,9 \mathrm{mg} \mathrm{dm}^{-3}$.

A calagem foi realizada no ano agrícola anterior, em agosto de 2006, e antecedeu o cultivo de milho, seguido por um cultivo de batata-doce, com dose de 1 t ha ${ }^{-1}$ de calcário dolomítico, de PRNT igual a $90 \%$. O preparo de solo se deu

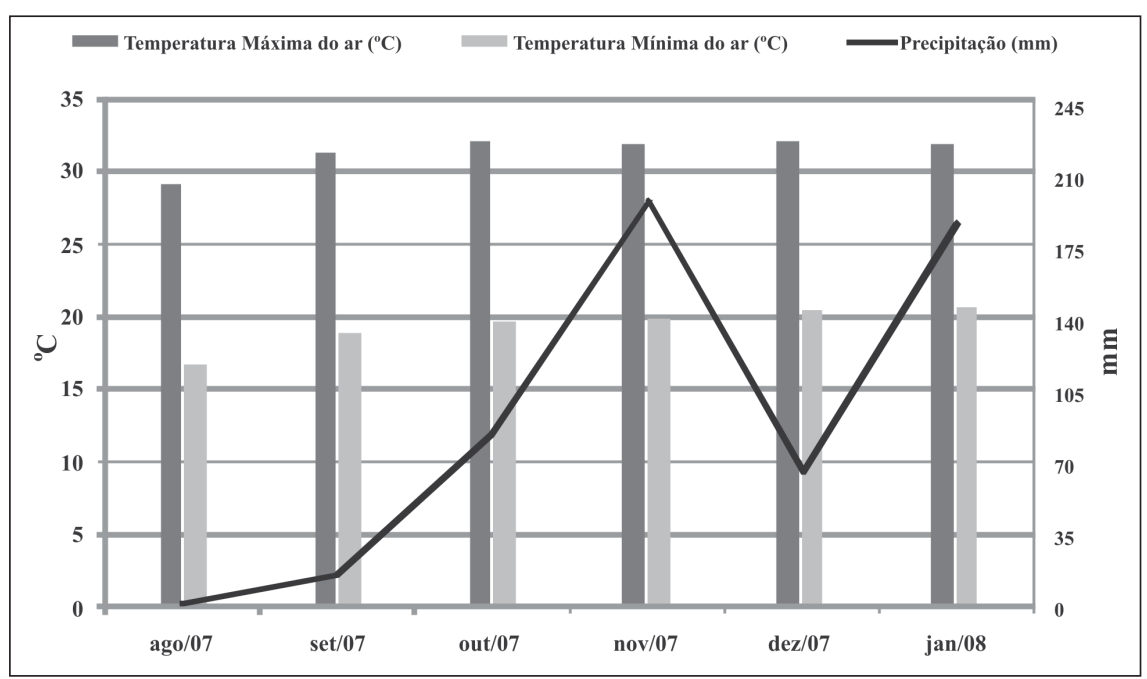

Figura 1: Temperatura máxima e mínima do ar e precipitação durante a condução do experimento. (maximum and minimum air temperature and precipitation during the conduction of the research). Presidente Prudente, UNOESTE, 2008.

com duas arações pesadas, três gradagens leves e com a marcação de curvas de níveis. As leiras foram demarcadas com espaçamento entre si de $0,90 \mathrm{~m}$. O transplantio foi realizado em meados de agosto de 2007, utilizando-se ramas da cultivar Canadense, coletadas na mesma propriedade. A adubação de plantio foi de $250 \mathrm{~kg} \mathrm{ha}^{-1}$ do formulado NPK 04-30-10.

O delineamento experimental foi inteiramente casualizado, com três repetições e os tratamentos consistiram nas épocas de coleta iniciadas em 20 de setembro de 2007 e finalizadas em 27 de dezembro de 2007, totalizando oito épocas. As coletas iniciaram aos 40 dias após o transplantio (DAT), em intervalos de 15 dias.

A coleta foi realizada aleatoriamente na lavoura de batata-doce, sendo retiradas três plantas completas por repetição. Em seguida as plantas foram levadas ao laboratório de tecidos vegetais da UNOESTE, onde foram lavadas e separadas em folhas, ramas, raízes tuberosas e raiz.

Com o auxílio de uma balança eletrônica de precisão as amostras tiveram sua massa fresca determinada e foram encaminhadas à estufa de circulação de ar forçada, à temperatura de $65^{\circ} \mathrm{C}$, durante 72 horas. Após esse período as amostras tiveram sua massa seca determinada e foram moídas para posterior análise química, seguindo metodologia de
Malavolta et al. (1997). Foram avaliados o nitrogênio $(\mathrm{N})$; fósforo $(\mathrm{P})$; potássio $(\mathrm{K})$; cálcio $(\mathrm{Ca})$; magnésio $(\mathrm{Mg})$; enxofre $(\mathrm{S})$; boro $(\mathrm{B})$; cobre $(\mathrm{Cu})$; manganês $(\mathrm{Mn})$; ferro (Fe) e zinco ( $\mathrm{Zn})$.

Os dados foram analisados por meio de regressão, sendo ajustadas equações significativas até $5 \%$ de probabilidade pelo teste $\mathrm{F}$ com os maiores coeficientes de determinação $\left(\mathrm{R}^{2}\right)$.

\section{RESULTADOS E DISCUSSÃO}

A maior produção de massa fresca entre os órgãos de batata-doce na colheita ocorreu nas ramas, com aproximadamente 40,4 $\mathrm{t} \mathrm{ha}^{-1}$, seguida pelas raízes tuberosas com $36,5 \mathrm{t} \mathrm{ha}^{-1}$, pelas folhas com 33,2 $\mathrm{t} \mathrm{ha}^{-1}$ e pelas raízes com 3,9 t ha $^{-1}$ (Figura 2a). Folhas e raízes tuberosas tiveram ajuste linear na produção de massa verde, enquanto as ramas e as raízes ajuste quadrático, sendo que para todos os órgãos a maior produção de massa fresca ocorreu por ocasião da colheita.

As massas secas das folhas e das raízes tuberosas tiveram crescimento linear positivo até os 115 DAT. O mesmo não ocorreu com os demais órgãos da planta, ramas e raiz, onde o ajuste foi quadrático (Figura 2b). Entre 115 e 130 DAT, houve um forte crescimento da massa seca de raízes tuberosas, reflexo da translocação e do acúmulo de nutrientes, sendo que após esse período hou- 


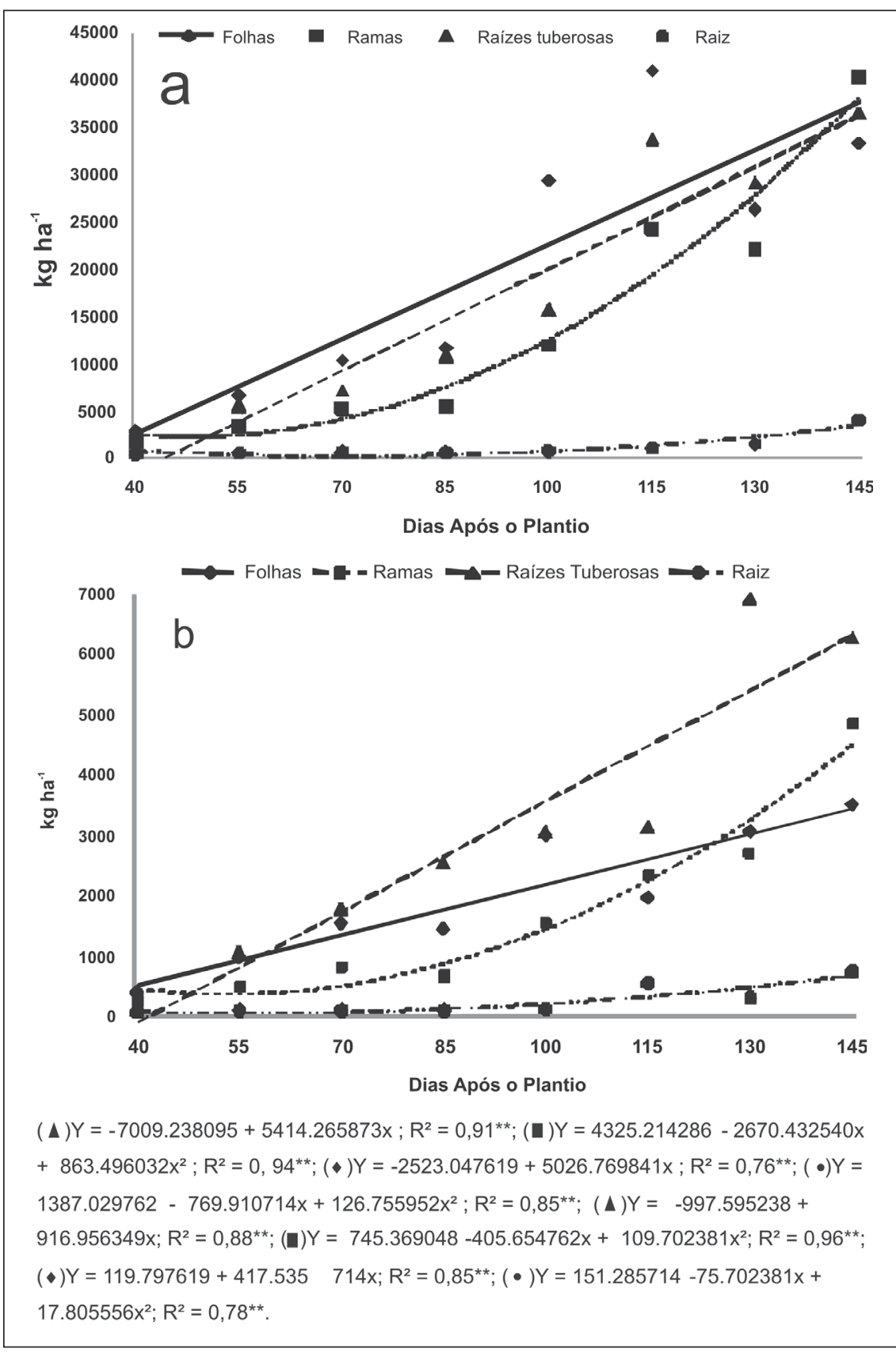

Figura 2. (a) Distribuição da massa fresca entre órgãos de batata-doce, cultivar Canadense, em lavoura comercial; (b) Distribuição da massa seca entre órgãos de batata-doce.((a) fresh matter distribution among sweet potato organs, cultivar Canadense, in commercial farming; (b) dry matter distribution among sweet potato organs). Presidente Prudente, UNOESTE, 2008.

** Significativo a $1 \%$.

ve diminuição na massa seca das raízes tuberosas. Esse fato se explica pelo ciclo perene da cultura, pois nessa fase a planta redireciona os fotoassimilados e investe no crescimento vegetativo (Hauman, 1992).

Esses resultados estão de acordo às curvas de acúmulo de massa seca de algumas espécies de olerícolas, que geralmente possuem três fases distintas: na primeira fase a absorção é lenta, se- guida de intensa absorção até atingir o ponto máximo, a partir do qual ocorre um pequeno declínio (Prata, 1999; Araújo et al., 2001; Lima, 2001).

Porém, no trabalho de Conceição et al. (2004), o pico de produção de massa seca de folhas e de ramas foi entre $110 \mathrm{e}$ 116 DAT para as cultivares Abóbora e Da Costa, e o acúmulo de massa seca foi diferente entre as duas cultivares, diferentemente dos resultados obtidos no presente trabalho, onde a maior produção de massa seca de folhas e ramas foi obtida por ocasião da colheita.

Já para a produção de massa seca de raízes tuberosas, Conceição et al. (2004) constataram que as cultivares responderam de maneira diferenciada, sendo que a cultivar Abóbora obteve maior quantidade de massa seca aos 130 DAT, enquanto a cultivar Da Costa aos 150 DAT. Existe certa semelhança entre a cultivar Abóbora e a Canadense, utilizadas neste ensaio, ambas com pico de produção de massa seca e de raízes tuberosas aos 130 DAT, com um pequeno declínio até o momento da colheita.

Na Figura 3 está a marcha de absorção de nutrientes pelas folhas de batata-doce, cv. Canadense e no Quadro 1 estão dispostas as respectivas equações ajustadas. Desde o início das amostragens, o nitrogênio (N) foi o macronutriente mineral mais absorvido pelas folhas de batata-doce, com taxa de absorção elevada até os 100 DAT, quando o teor absorvido pelas folhas diminuiu aos 115 DAT, voltando a aumentar até a colheita, aos 145 DAT, com um total de $124 \mathrm{~kg} \mathrm{ha}^{-1}$ de $\mathrm{N}$ extraído pelas folhas.

O segundo macronutriente em ordem de extração pelas folhas de batata-doce foi o $\mathrm{K}$, com comportamento semelhante ao N, chegando a exportar cerca de 83 $\mathrm{kg} \mathrm{ha}^{-1}$ de $\mathrm{K}$ na época da colheita. O Ca foi responsável pela terceira maior taxa de absorção pelas folhas de batata-doce, com extração de $52 \mathrm{~kg} \mathrm{ha}^{-1}$ de Ca por ocasião da colheita. Os demais nutrientes, $\mathrm{Mg}, \mathrm{S}$, e P tiveram menores taxas de absorção pelas folhas no momento da colheita, com valores de 17; 15 e $10 \mathrm{~kg}$ $\mathrm{ha}^{-1}$, respectivamente. Esses resultados obtidos na cultura da batata-doce, divergem de outros autores em outras culturas, como por Fernandes et al (1971) que avaliaram a marcha de absorção de nutrientes em folhas de alface e obtiveram a seguinte ordem de extração em plantas com 65 dias: $\mathrm{K}>\mathrm{N}>\mathrm{Ca}>\mathrm{P}>\mathrm{Mg}>\mathrm{S}$. No estudo de Furlani et al (1978), avaliou-se a extração de nutrientes nas folhas de olerícolas tuberosas como a cenoura e a beterraba, e concluiu-se que a ordem de extração foi: $\mathrm{K}>\mathrm{N}>\mathrm{Ca}>\mathrm{S}>\mathrm{Mg}>\mathrm{P} \quad \mathrm{e}$ $\mathrm{K}>\mathrm{N}>\mathrm{Mg}>\mathrm{Ca}>\mathrm{P}>\mathrm{S}$, respectivamente. 
Em termos de extração de micronutrientes pelas folhas de batatadoce, o Mn foi o mais absorvido, com exportação de $380 \mathrm{~g} \mathrm{ha}^{-1}$ no momento da colheita, aos 145 DAT (Figura 3). Em seguida tem-se o $\mathrm{B}, \mathrm{Zn}, \mathrm{Fe}$, e $\mathrm{Cu}$, com extrações de $162 ; 87 ; 48$ e $41 \mathrm{~g} \mathrm{ha}^{-1}$, respectivamente. A marcha de absorção de micronutrientes nas culturas da beterraba e cenoura teve a seguinte ordem de extração, para ambas as culturas: $\mathrm{Fe}>\mathrm{Mn}>\mathrm{Zn}>\mathrm{B}>\mathrm{Cu}$ (Furlani et al, 1978), no entanto no estudo de Fernandes et al (1971) o Mn foi o micronutriente mais absorvido, seguido pelo $\mathrm{Zn}$, Fe e $\mathrm{Cu}$.

Nas ramas, assim como nas folhas, $\mathrm{o}$ $\mathrm{N}$ foi o nutriente mais absorvido, com extração de $85 \mathrm{~kg} \mathrm{ha}^{-1}$, aos 145 DAT (Figura 3). Por sua vez, o Ca foi o segundo nutriente em ordem de extração, perfazendo um total de $75 \mathrm{~kg} \mathrm{ha}^{-1}$ nas ramas de batata-doce no momento da colheita, seguido pelo K com $56 \mathrm{~kg} \mathrm{ha}^{-1}$, pelo $\mathrm{Mg}$ com $16 \mathrm{~kg} \mathrm{ha}^{-1}, \mathrm{~S}$ com 12,8 $\mathrm{kg} \mathrm{ha}^{-1}$ e P com $11,8 \mathrm{~kg} \mathrm{ha}^{-1}$. Todos os nutrientes apresentaram altos coeficientes de determinação no ajuste das curvas de extração de macro e micronutrientes nas ramas, conforme o Quadro 1. No trabalho de Espíndola et al (1998), que avaliou a influência da adubação verde sobre o acúmulo de N, P e K nas ramas e nas raízes tuberosas da batata-doce, concluiu-se que o $\mathrm{K}$ foi o nutriente mais extraído, seguido pelo $\mathrm{N}$ e pelo $\mathrm{P}$.

De acordo com a Figura 3, a extração de micronutrientes nas ramas de batatadoce teve o Mn como o micronutriente mais extraído com $162 \mathrm{~g} \mathrm{ha}^{-1}$, seguido pelo B com $114 \mathrm{~g} \mathrm{ha}^{-1}$, pelo Fe com $65 \mathrm{~g}$ ha $^{-1}$, pelo $\mathrm{Zn}$ com $57 \mathrm{~g} \mathrm{ha}^{-1}$ e pelo $\mathrm{Cu}$ com $41 \mathrm{~g} \mathrm{ha}^{-1}$.

Os resultados da marcha de absorção de macronutrientes revelam que o Ca foi o nutriente extraído em maior quantidade pelas raízes de batata-doce (Figura 3), com extração de $25 \mathrm{~kg} \mathrm{ha}^{-1}$ no momento da colheita. De acordo com Malavolta et al (1974), uma das principais funções do $\mathrm{Ca}$, juntamente com o $\mathrm{B}$, é o crescimento, desenvolvimento e o funcionamento do sistema radicular, sendo essencial na divisão celular (mitose). Os demais nutrientes, em ordem de extração por ocasião da colheita foram N, K, P, Mg e S com 12; 5,6; 1,5; 1,5 e $1,2 \mathrm{~kg} \mathrm{ha}^{-1}$, respectivamente. De

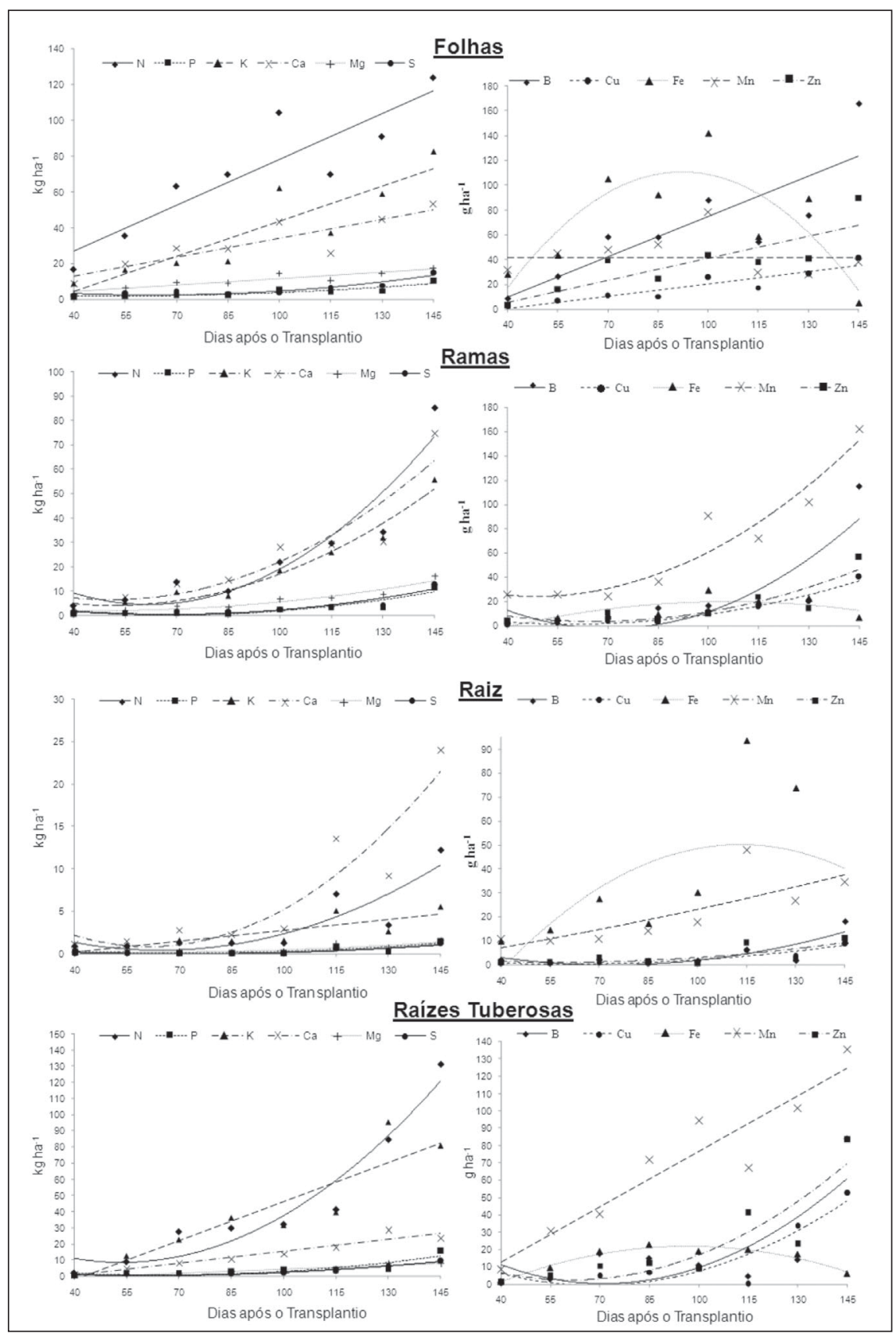

Figura 3. Marcha de absorção de macronutrientes e de micronutrientes pelas folhas ${ }^{1}$; $\operatorname{ramas}^{2}$; raíz e pelas raízes tuberosas ${ }^{2}$ de batata-doce, cultivar Canadense (absorption march of macro and micronutrients in leaves, stems, root and storage root of sweet potato, cultivar Canadense). Presidente Prudente, UNOESTE, 2008.

${ }^{1}$ Os valores reais de $\mathrm{Fe}$ e $\mathrm{Mn}$ foram divididos por 10, obtendo-se os valores do gráfico; ${ }^{2}$ Valores de $\mathrm{Fe}$ foram divididos por 10 ( ${ }^{1}$ the real values for $\mathrm{Fe}$ and $\mathrm{M}^{\mathrm{n}}$ were divided for 10 , obtaining the values of the figure; ${ }^{2}$ values of Fe were divided for 10).

acordo com o ajuste das curvas de extração de nutrientes pela raiz, o $\mathrm{K}$ e o $\mathrm{Mn}$ foram os únicos nutrientes onde o ajuste foi linear, os demais tiveram ajuste quadrático (Quadro 1).

Furlani et al (1978) estudaram a composição mineral da raiz de culturas produtoras de raízes tuberosas e concluiram que a ordem de extração da beterraba foi: $\mathrm{K}>\mathrm{N}>\mathrm{P}>\mathrm{Mg}>\mathrm{Ca}>\mathrm{S}$; já a cenoura (cultivar Nantes) apresentou a seguinte ordem: $\mathrm{K}>\mathrm{N}>\mathrm{Ca}>\mathrm{P}>\mathrm{Mg}>\mathrm{S}$ e o nabo e o rabanete apresentaram a mesma ordem de extração de macronutrientes pela raiz: $\mathrm{K}>\mathrm{N}>\mathrm{P}>\mathrm{S}>\mathrm{Ca}>\mathrm{Mg}$.

Assim, observa-se que a batata-doce apresenta comportamento diferenciado de absorção de nutrientes pela raiz, quan- 
do comparada a outras olerícolas produtoras de raízes tuberosas, onde a extração de Ca varia da terceira à quinta posição entre os macronutrientes.

Na Figura 3, tem-se a ordem de extração de micronutrientes pela raiz da batata-doce: $\mathrm{Mn}>\mathrm{B}>\mathrm{Zn}>\mathrm{Fe}>\mathrm{Cu}$, com taxas de $35 ; 18 ; 10,9 ; 9,9$ e 8,93 $\mathrm{g} \mathrm{ha}^{-1}$, respectivamente. Esses resultados são diferentes dos observados por Furlani et al (1978) na marcha de absorção de micronutrientes em culturas olerícolas produtoras de raízes como a cenoura (cultivar Nantes): $\mathrm{Fe}>\mathrm{Mn}>\mathrm{Zn}>\mathrm{B}>\mathrm{Cu}$ e pela beterraba, nabo e rabanete, que se assemelharam de extração de nutrientes: $\mathrm{Fe}>\mathrm{Zn}>\mathrm{B}>\mathrm{Mn}>\mathrm{Cu}$.

$\mathrm{O} \mathrm{N}$ foi o nutriente mais absorvido pelas raízes tuberosas, com taxa de 129 $\mathrm{kg} \mathrm{ha}^{-1}$, com o maior período de absorção entre 115-145 DAT; seguido pelo K com $81 \mathrm{~kg} \mathrm{ha}^{-1}$, que apresentou aos 115 130 DAT a maior taxa de absorção e pelo Ca com $23 \mathrm{~kg} \mathrm{ha}^{-1}$ (Figura 3). Os demais macronutrientes foram extraídos a taxas de 16; 9,6 e 7,4 $\mathrm{kg} \mathrm{ha}^{-1}$ para $\mathrm{P}, \mathrm{S}$ e $\mathrm{Mg}$, respectivamente. Entre os micronutrientes, o Mn foi o mais extraído com $136 \mathrm{~g} \mathrm{ha}^{-1}$; seguido do B com taxa de $84 \mathrm{~g} \mathrm{ha}^{-1}$, e do $\mathrm{Zn}$ com $82 \mathrm{~g} \mathrm{ha}^{-1}$. Os demais (Fe e $\mathrm{Cu}$ ) apresentaram absorção de 61 e $52 \mathrm{~g} \mathrm{ha}^{-1}$, respectivamente (Figura 3).

De acordo com o Quadro 1, para o K, $\mathrm{Ca}, \mathrm{Mg}$ e Mn foram ajustadas curvas de extração lineares, enquanto para o N, P, $\mathrm{S}, \mathrm{B}, \mathrm{Fe}, \mathrm{Cu}$ e $\mathrm{Zn}$ o ajuste foi quadrático.

A Tabela 1 mostra a marcha de absorção de nutrientes pelos órgãos da batata-doce no momento da colheita. A maior parte dos nutrientes absorvidos está nas folhas, ramas e nas raízes tuberosas.

As raízes tuberosas representam a grande fonte de exportação de nutrientes pela batata-doce, e para essa produtividade de $6.290 \mathrm{~kg} \mathrm{ha}^{-1}$ de massa seca, os macronutrientes mais extraídos foram o N com $129 \mathrm{~kg} \mathrm{ha}^{-1}$ e o K com $81 \mathrm{~kg} \mathrm{ha}^{-1}$, mostrando assim que boa parte dos nutrientes extraídos está contida nas raízes tuberosas, principal dreno de assimilados da planta, analogamente aos frutos na cultura da abóbora (Vidigal et al., 2007); no tomate (Fayad et al.,2001), e na cultura da melancia (Grangeiro \& Cecílio Filho, 2005).

Quadro 1. Equações de regressão para a marcha de absorção de nutrientes pelos órgãos de batata-doce, cultivar Canadense (Regression equations for absorption march of nutrients by sweet potato organs, Cultivar Canadense). Presidente Prudente, UNOESTE, 2008.

\begin{tabular}{|c|c|c|}
\hline & Folhas & Ramas \\
\hline N & $\begin{array}{l}Y=13,910095+12,867794 x ; R^{2}= \\
0,81^{* *}\end{array}$ & $\begin{array}{l}Y=17,953976-10,987012 x+ \\
2,237702 x^{2} ; R^{2}=0,89^{* *}\end{array}$ \\
\hline$P$ & $\begin{array}{l}Y=2,43676-0,639484 x+0,184897 x^{2} ; R^{2} \\
=0,81^{* *}\end{array}$ & $\begin{array}{l}Y=3,548131-4,347276 x+1,230812 x^{2} \\
R^{2}=0,83^{* *}\end{array}$ \\
\hline K & $Y=-5,312726+9,771782 x ; R^{2}=0,81^{* *}$ & $\begin{array}{l}Y=7,831839-4,347276 x+1,230812 x^{2} \\
R^{2}=0,96^{\star *}\end{array}$ \\
\hline $\mathrm{Ca}$ & $Y=7,579190+5,308198 x ; R^{2}=0,78^{* *}$ & $\begin{array}{l}Y=10,532923-4,984073 x+1,450554 x^{2} \\
R^{2}=0,86^{\star *}\end{array}$ \\
\hline $\mathrm{Mg}$ & $Y=2,530262+1,820377 x ; R^{2}=0,87^{* *}$ & $\begin{array}{l}Y=2,370625-0,862192 x+0,295998 x^{2} \\
R^{2}=0,93^{* *}\end{array}$ \\
\hline S & $\begin{array}{l}Y=4,9173-1,940058 x+0,376355 x^{2} ; R^{2} \\
=0,87^{* *}\end{array}$ & $\begin{array}{l}Y=3,541810-2,261500 x+0,395190 x^{2} \\
R^{2}=0,85^{\star *}\end{array}$ \\
\hline B & $\begin{array}{l}Y=-5,113476+16,077948 x ; R^{2}= \\
0,70^{\star *}\end{array}$ & $\begin{array}{l}Y=31,663244-22,399018 x+ \\
3,685204 x^{2} ; R^{2}=0,74^{\star *}\end{array}$ \\
\hline $\mathrm{Cu}$ & $Y=-4,438179+4,980623 x ; R^{2}=0,85^{\star *}$ & $\begin{array}{l}Y=6,491048-4,786190 x+1,074087 x^{2} \\
R^{2}=0,95^{\star \star}\end{array}$ \\
\hline $\mathrm{Fe}$ & $\begin{array}{l}Y=-440,15+690,54 x-77,02 x^{2} ; R^{2}= \\
0,71^{* *}\end{array}$ & $\begin{array}{l}Y=-110,97+114,32 x-10,626 x^{2} ; R^{2}= \\
0,53^{* *}\end{array}$ \\
\hline $\mathrm{Mn}$ & $Y=438,4 ; n s$ & $\begin{array}{l}Y=32,130952-9,837302 x+3,123016 x^{2} \\
R^{2}=0,91^{* *}\end{array}$ \\
\hline \multirow[t]{2}{*}{$\mathrm{Zn}$} & $Y=-0,011905+8,373016 x ; R^{2}=0,70^{* *}$ & $\begin{array}{l}Y=14,821429-8,527778 x+1,567460 x^{2} \\
R^{2}=0,77^{\star *}\end{array}$ \\
\hline & Raiz & Raízes Tuberosas \\
\hline$N$ & $\begin{array}{l}Y=2,8948-1,848609 x+0,350185 x^{2} ; R^{2} \\
=0,77^{\star *}\end{array}$ & $\begin{array}{l}Y=19,132726-11,523865 x+ \\
3,033667 x^{2} ; R^{2}=0,93^{* *}\end{array}$ \\
\hline$P$ & $\begin{array}{l}Y=0,5081-0,297018 x+0,048696 x^{2} ; R^{2} \\
=0,70^{* *}\end{array}$ & $\begin{array}{l}Y=3,787994-2,270534 x+0,420665 x^{2} \\
R^{2}=0,77^{\star *}\end{array}$ \\
\hline K & $Y=-0,448857+0,640579 x ; R^{2}=0,68^{* *}$ & $\begin{array}{l}Y=-14156012+12,089206 x ; R^{2}= \\
0,83^{\star *}\end{array}$ \\
\hline $\mathrm{Ca}$ & $\begin{array}{l}Y=4,8840-3,329187 x+0,679917 x^{2} ; R^{2} \\
=0,85^{* *}\end{array}$ & $Y=-3,126238+3,679933 x ; R^{2}=0,91^{* *}$ \\
\hline $\mathrm{Mg}$ & $\begin{array}{l}Y=0,3082-0,154540 x+0,036698 x^{2} ; R^{2} \\
=0,78^{* *}\end{array}$ & $Y=-1,534381+1,162075 x ; R^{2}=0,92^{* *}$ \\
\hline$S$ & $\begin{array}{l}Y=0,4107-0,257893 x+0,042464 x^{2} ; R^{2} \\
=0,77^{* *}\end{array}$ & $\begin{array}{l}Y=2,032417-1,322786 x+0,274063 x^{2} \\
R^{2}=0,95^{\star *}\end{array}$ \\
\hline B & $\begin{array}{l}Y=6,3848-3,890921 x+0,601944 x^{2} ; R^{2} \\
=0,68^{\star *}\end{array}$ & $\begin{array}{l}Y=24,057821-15,307901 x+ \\
2,486940 x^{2} ; R^{2}=0,61^{* *}\end{array}$ \\
\hline $\mathrm{Cu}$ & $\begin{array}{l}Y=1,5605-1,122567 x+0,244774 x^{2} ; R^{2} \\
=0,82^{* *}\end{array}$ & $\begin{array}{l}Y=11,368036-8,540095 x+ \\
1,669921 x^{2} ; R^{2}=0,95^{* *}\end{array}$ \\
\hline $\mathrm{Fe}$ & $\begin{array}{l}Y=-26,059+25,964 x-2,2103 x^{2} ; R^{2}= \\
0,33\end{array}$ & $\begin{array}{l}Y=-109,636+138,0456 x-14,4226 x^{2} ; R^{2} \\
=0,94^{* *}\end{array}$ \\
\hline $\mathrm{Mn}$ & $Y=1,904762+4,373016 x ; R^{2}=0,60^{* *}$ & $\begin{array}{l}Y=-3,583333+16,027778 x ; R^{2}= \\
0,89^{\star *}\end{array}$ \\
\hline $\mathrm{Zn}$ & $\begin{array}{l}Y=2,3214-1,095238 x+0,246032 x^{2} ; R^{2} \\
=0,55^{\star *}\end{array}$ & $\begin{array}{l}Y=15,1547-10,3928 x+2,154762 x^{2} ; R^{2} \\
=0,79^{\star *}\end{array}$ \\
\hline
\end{tabular}

**Significativo a $1 \%$ de probabilidade pelo teste $\mathrm{F}$; $\mathrm{ns}=$ não significativo.

A ordem de extração de nutrientes pela planta de batata-doce foi: $\mathrm{N}>\mathrm{K}>\mathrm{Ca}>\mathrm{Mg}>\mathrm{P}>\mathrm{S}>\mathrm{Mn}>\mathrm{B}>\mathrm{Zn}>\mathrm{Fe}>\mathrm{Cu}$. Esses resultados corroboram com os obtidos por Pacheco et al (2005) que observaram maior extração de $\mathrm{N}$ que de $\mathrm{K}$ pela planta de abóbora cultivada em casa de vegetação. No entanto discordam dos resultados obtidos por Fontes \& Lima (1993), que observaram maior extração de potássio por plantas de abóbora e pepino e por Vidigal et al. (2007), também na cultura da abóbora, que observaram que a ordem de extração foi $\mathrm{K}>\mathrm{N}>\mathrm{Ca}>\mathrm{P}>\mathrm{Mg}>\mathrm{S}$.

O mesmo comportamento foi verificado por outros autores, em cucurbitáceas por Lima (2001) na cultura do melão; Solis et al. (1988) em pepino; Araújo et al. (2001) em abobrinha e 
Tabela 1. Marcha de absorção de nutrientes e produção de massa seca pelos órgãos de batata-doce, cultivar Canadense, por ocasião da colheita (absorption march of nutrients and dry mass production of sweet potato organs, cultivar Canadense, after harvest). Presidente Prudente, UNOESTE, 2008.

\begin{tabular}{|c|c|c|c|c|c|c|c|c|c|c|c|c|}
\hline \multirow{3}{*}{ Órgão } & \multirow{3}{*}{$\begin{array}{c}\text { MS } \\
\left(\mathrm{kg} \mathrm{ha}^{-1}\right)\end{array}$} & \multicolumn{11}{|c|}{ Absorção } \\
\hline & & \multicolumn{6}{|c|}{$\mathrm{kg} \mathrm{ha}^{-1}$} & \multicolumn{5}{|c|}{$\mathrm{g} \mathrm{ha}^{-1}$} \\
\hline & & $\mathbf{N}$ & $\mathbf{P}$ & $\mathrm{K}$ & $\mathrm{Ca}$ & $\mathrm{Mg}$ & $\mathrm{S}$ & B & $\mathrm{Cu}$ & $\mathrm{Fe}$ & Mn & $\mathrm{Zn}$ \\
\hline Folhas & 3525,0 & 124,0 & 11,0 & 83,0 & 52,0 & 17,0 & 15,0 & 162,0 & 41,0 & 48,0 & 380,0 & 87,0 \\
\hline Ramas & 4870,0 & 85,0 & 12,0 & 56,0 & 75,0 & 16,0 & 13,0 & 114,0 & 41,0 & 65,0 & 162,0 & 57,0 \\
\hline Raiz & 744,7 & 12,0 & 1,5 & 5,6 & 24,0 & 1,5 & 1,2 & 18,0 & 9,0 & 10,0 & 35,0 & 11,0 \\
\hline Raiz tuberosa* & 6290,0 & 129,0 & 16,0 & 81,0 & 23,0 & 7,4 & 9,6 & 84,0 & 52,0 & 61,0 & 136,0 & 82,0 \\
\hline Total & $15.429,7$ & 350,0 & 40,5 & 225,6 & 174,0 & 41,9 & 38,8 & 378,0 & 143,0 & 184,0 & 713,0 & 237,0 \\
\hline
\end{tabular}

*Exportado pela colheita das raízes tuberosas (*exported from storage roots after harvest); MS= massa seca (MS= dry mass).

por Grangeiro \& Cecílio Filho (2005) em melancia. Na cultura da berinjela, Malavolta et al (1974) ordenam os nutrientes na seguinte ordem de extração: $\mathrm{K}>\mathrm{N}>\mathrm{Ca}>\mathrm{Mg}>\mathrm{P}>\mathrm{S}$.

Dentre os nutrientes absorvidos pela batata-doce destaca-se a elevada absorção de $\mathrm{N}$, o que pode ser explicado pela associação da batata-doce com microorganismos do solo, já que foram fornecidos apenas $10 \mathrm{~kg} \mathrm{ha}^{-1}$ de $\mathrm{N}$ via fertilizante no momento do plantio e, o teor de matéria orgânica do solo, importante fonte de $\mathrm{N}$ às plantas, foi relativamente baixo, de $10 \mathrm{~g} \mathrm{dm}^{-3}$. Byju (2008) recomenda a aplicação de $10 \mathrm{~kg} \mathrm{ha}^{-1} \mathrm{de}$ Azospirillum $^{\circledR}$; nesse caso a dose de $\mathrm{N}$ pode ser reduzida em até $35 \%$, pois a associação com esses microorganismos resulta em maior produção de fitohormônios, o que acarreta em maior crescimento da planta.

De acordo com Lorenzi et al (1997), a recomendação oficial de adubação nitrogenada na cultura da batata-doce no estado de São Paulo é de $20 \mathrm{~kg} \mathrm{ha}^{-1}$ de $\mathrm{N}$ no plantio, e de $30 \mathrm{~kg} \mathrm{ha}^{-1}$ de $\mathrm{N}$ em cobertura, totalizando $50 \mathrm{~kg} \mathrm{ha}^{-1} \mathrm{de} \mathrm{N}, \mathrm{o}$ que, caso fosse a única fonte de $\mathrm{N}$ à cultura, seria de apenas $15 \%$ da necessidade da batata-doce.

A produção de massa seca dos órgãos de batata-doce apresentou a ordem: raízes tuberosas, ramas, folhas e raiz. $\mathrm{O} \mathrm{N}$ e o $\mathrm{Mn}$ foram os macro e micronutrientes mais absorvidos pelas folhas, ramas e raízes tuberosas de batata-doce. Na raiz, observou-se maior absorção dos macro e micronutrientes $\mathrm{Ca}$ e Mn, respectivamente e, os períodos de maior exigência de $\mathrm{N}$ e $\mathrm{K}$ pelas raízes tuberosas foi entre os 115-145 e 115-130 DAT, nesta ordem.

\section{AGRADECIMENTOS}

Ao proprietário e aos funcionários do Rancho Ebenezer, Marcos e Ricardo por terem cedido a área para realização do experimento e pela colaboração na condução do mesmo.

\section{REFERÊNCIAS}

ARAÚJO WF; BOTREL TA; CARMELLO QAC; SAMPAIO RA; VASCONCELOS MRB. 2001. Marcha de absorção de nutrientes pela cultura da abobrinha conduzida sob fertirrigação. In: FOLEGATTI MV; CASARINI E; BLANCO FF; BRASIL RPC; RESENDE RS. (Cood.) Fertirrigação: flores, frutas e hortaliças. Guaíba: Agropecuária: 1: 67-77.

BYJU G. 2008, 28 março. Sweet potato. Disponível em http://www.ctcri.org/ byju.htmhttp

CONCEIÇÃO MK; LOPES NF; FORTES GRL. 2004. Partição de matéria seca entre órgãos de batata-doce (Ipomoea batatas (L.) Lam), cultivares Abóbora e Da Costa. Revista Brasileira de Agrociência 10: 313-316.

EMBRAPA. Empresa Brasileira de Pesquisa Agropecuária. 2008, 20 de março. Cultura da batata-doce. Sistemas de produção, versão eletrônica. Disponível em: http:// www.cnph.embrapa.br/cultivares/batdoce.htm

EMBRAPA. Centro Nacional de Pesquisa de Solos (Rio de Janeiro-RJ). 1999. Sistema Brasileiro de Classificação de Solos. Brasília: Embrapa-SPI, Embrapa-CNPS, $412 \mathrm{p}$.

ESPINDOLA JAZ; ALMEIDA DL; GUERRA JGM; SILVA EMR; SOUZA, FA 1998. Influencia da adubação verde na colonização micorrízica e na produção de batata-doce. Pesquisa Agropecuária Brasileira 33: 339 347.

FABRO LE; BAUTISTA OK; MALIXI MM. 1976. Dry matter accumulation and nutrient uptake of "BNAS 51" sweet potato as different stages of growth. The Philippine Agriculturist. 60: 5-6.
FAGERIA NK; BALIGAR VC. 1993. Screening crop genotypes for mineral stresses. In: WORKSHOP ON ADAPTATION OF PLANTS TO SOIL STRESS. Linclon, 1993. Proceedings. Linclon, University of Nebraska. p.142159.

FAYAD JA; FONTES PCR; CARDOSO AA; FINGER LF; FERREIRA FA. 2001. Crescimento e produção do tomateiro cultivado sob condições de campo e de ambiente protegido. Horticultura brasileira 19: 365-370.

FERREIRA ME; CASTELLANE PD; CRUZ MCP. 1990. Nutrição e adubação de hortaliças. Simpósio sobre nutrição e adubação de hortaliças, Jaboticabal-SP: Potafós. 480p.

FERNANDES PD; OLIVEIRA GD; HAAG HP. 1971. XI Reunião Anual da Sociedade de Olericultura do Brasil, Piracicaba.

FONTES RR; LIMA JA. Nutrição mineral e adubação do pepino e da abóbora. In: FERREIRA ME; CASTELLANE PD; CRUZ MCP. Nutrição e adubação de hortaliças. Jaboticabal-SP: Potafós, p. 281300.

FURLANI AMC; FURLANI PR; BATAGLIA OC; HIROCE R; GALLO JR; BERNARDI JB; FORNASIER JB; CAMPOS HR. 1978. Composição mineral de diversas hortaliças. Bragantia 37: 33-44.

GRANGEIRO LC; CECÍLIO FILHO AB. 2005. Acúmulo e exportação de macronutrientes em melancia sem sementes. Horticultura brasileira 23: 763-767.

HUAMAN Z. 1992. Systemic botany and morphology of the sweetpotato plant. Technical Information Bulletin 25. International Potato Centre, Lima, Peru. $22 \mathrm{p}$.

HAHN SK. 1977. Sweet potato. In: ALVIM PT; KOZLOWSKI TT. (eds). Ecophysiology of tropical crops. New York: Academic Press. p. 237-248.

LIMA AA. 2001. Absorção e eficiência de utilização de nutrientes por híbridos de melão (Cucumis melo L.). Fortaleza: UFC. 60 p. (Tese mestrado).

MALAVOLTA EA; VITTI GC; OLIVEIRA AS. 1997. Avaliação do estado nutricional das plantas: princípios e aplicações. Piracicaba: Potafós. 201p. 
MALAVOLTA E; HAAG HP; MELLO FAF; BRASIL SOBRINHO MOC. 1974. Nutrição mineral e adubação de plantas cultivadas. São Paulo, Livr. Pioneira, 727p.

MARSCHNER H. 1995. Mineral nutrition of higher plants. London: Academic Press. $889 \mathrm{p}$.

PACHECO DD; VIDIGAL SM; MOREIRA SAF; SOUZA FV; LIMA LMS; MARTINS FG; DIAS WOB; MOREIRA LLQ. 2005. Crescimento e marcha de absorção de macronutrientes para abóbora híbrida cultivada em casa de vegetação. In: CONGRESSO BRASILEIRO DE OLERICULTURA, 45. Anais... Fortaleza: $\mathrm{ABH}$.
PRATA EB. 1999. Acumulação de biomassa e absorção de nutrientes por híbridos de meloeiro (Cucumis melo L.). 1999. Fortaleza: UFC. 37p. (Tese mestrado).

SCOTT LE. 1950. Potassium uptake by the sweet potato plant. Proceedings of the American Society of Horticultural Science 56: 248-252.

SILVA JBC; LOPES CA; MAGALHÃES JS. 2002. Cultura da batata-doce. In: CEREDA MP; Agricultura: Tuberosas amiláceas latino americanas, São Paulo: Cargill, 2: 449-503.
SOLIS FAM; HAAG HP; MINAMI K; DIEHL WJ. 1988. Nutrição de hortaliças. LVI Acumulação de nutrientes na cultura do pepino (Cucumis sativus L.) var. Aodai cultivado em condições de campo. Anais da Escola Superior de Agricultura Luiz de Queiroz 39: 697-737.

VIDIGAL SM; PACHECO DD; FACION CE. 2007. Crescimento e acúmulo de nutrientes pela abóbora híbrida tipo Tetsukabuto. Horticultura Brasileira 25: 375-380. 